\title{
Estructura y propósitos comunicativos en Tesis de Magíster y Licenciatura*
}

\author{
Sofía Zamora** \\ René Venegas***
}

\begin{abstract}
Resumen
El presente artículo aborda el estudio del género tesis desde la perspectiva estructural y funcional del estudio de los textos. De esta manera, el objetivo de esta investigación es describir la superestructura y los propósitos comunicativos de tesis de Lingüística y Filosofía en los niveles de Licenciatura y Magíster. La metodología consistió en aplicar categorías existentes, así como también emergentes para la sistematización de los hallazgos. El corpus estuvo compuesto por 20 tesis del corpus TFGPUCV-2010 (5 tesis en cada disciplina y nivel). Los resultados indican que en cuanto a la superestructura textual, existe diferencia según la disciplina, mientras que en relación a los propósitos comunicativos se establecen diferencias según la disciplina y el nivel, destacándose el grado de explicitud en la identificación al propósito comunicativo de cada apartado de la superestructura. Por último, es posible concluir que el género tesis no es homogéneo y que su variación se relaciona más con la disciplina que con el grado académico, de acuerdo a las necesidades de las comunidades discursivas particulares.
\end{abstract}

Palabras clave: tesis, superestructura, propósito comunicativo

\section{Structure and communicative purposes in Master and Undergraduate Thesis}

\begin{abstract}
The paper approaches the study of the thesis genre from a functional and structural perspective. The aim of this research is to identify communicative purposes and to describe the superstructure of linguistics and philosophy thesis, written by undergraduate and master students. The methodology consisted in the application of existing, as well emergent categories to a corpus made up of 20 theses collected from the TFGPUCV-2010 Corpus ( 5 theses from each discipline and level). According to the findings, there are differences regarding the superstructure depending on the discipline. In respect to the communicative purposes there differences between discipline and level can be observed. The explicitness of the communicative purpose of each section of the superstructure varies. According to our results, we conclude that the thesis genre is not homogeneous and varies according to the disciplines more than the academic degree, responding to the necessities of particular discourse communities.
\end{abstract}

Keywords: thesis, superestructure, ommunicative purpose

Recibido: 30-03-2012

Aceptado: 31-10-2012

* Artículo vinculado al proyecto Fondecyt N No 1101039 Caracterización del macro género trabajo final de grado en licenciatura y magíster: desde los patrones léxico-gramaticales y retórico-estructurales al andamiaje de la escritura acdémica.

* Licenciada en Lengua y Literatura Hispánicas. Pontificia Universidad Católica de Valparaíso, Valparaíso, Chile. sofia.zamora.h@gmail.com

*** Dr. en Lingüística. Pontificia Universidad Católica de Valparaíso, Valparaíso, Chile. rene.venegas@ ucv.cl 


\section{Introducción}

En el ámbito de los estudios del lenguaje en uso, surgen diversas perspectivas de análisis de los aspectos que constituyen a los textos como manifestaciones del lenguaje situado. De ellas emergen los géneros discursivos, entendidos como formas de uso del lenguaje comprendidos por un conjunto de enunciados estables y marcados por una estilística, una estructura y una intencionalidad determinada, según la esfera de actividad social de los hablantes en la que se circunscriben (Bajtin, 1999).

Dos de estas particularidades de los géneros discursivos propuestas por Bajtin (1999) dicen relación con los estudios estructurales del lenguaje, concentrados en abordar la organización constitutiva de los textos; y los estudios funcionales, que tienen como foco el estudio del papel que los textos desempeñan como manifestación social en su contexto (Parodi, 2008). Uno de los aspectos que sustentan este primer enfoque es el estudio de la superestructura textual (van Dijk, 1998), es decir, el modo en que estos se organizan, generalmente, por medio de apartados. Desde la perspectiva del análisis funcional, emerge la noción de propósito comunicativo (Askehave \& Swales, 2001). Esta perspectiva enfatizará la identificación de la finalidad específica de los apartados constitutivos de la estructura organizacional de un texto.

Por otra parte, en los últimos años, se ha puesto énfasis en los estudios de la escritura académica, caracterizando las producciones de cada comunidad discursiva y considerando las convenciones propias acordadas por los miembros de una comunidad. Estas convenciones permiten la existencia de géneros académicos característicos de las comunidades discursivas presentes en la esfera social (Parodi, 2008). Uno de estos géneros académicos lo constituye el Trabajo Final de Grado, entendido como aquel texto destinado a acreditar el conocimiento de un estudiante con el fin de obtener algún grado académico (Venegas, 2010). Esta área de estudio ha sido poco estudiada en español, lo que fundamenta la necesidad de una descripción estructural y funcional de estos géneros, con el fin de generar propuestas descriptivas y didácticas para la escritura de este género.

En este sentido, nos interesa caracterizar las tesis con relación a sus aspectos estructurales y funcionales, como una perspectiva integradora que nos permitirá realizar en mayor profundidad la caracterización de 
este género. Ante ello, el objetivo de esta investigación fue describir la superestructura y los propósitos comunicativos asociados a cada apartado de la organización, en las tesis de dos disciplinas: Lingüística y Filosofía; y en dos niveles académicos: Licenciatura y Magíster.

A continuación presentaremos, en primer lugar, los antecedentes teóricos que sustentaron nuestra investigación; en segundo término, se indicarán las características metodológicas de la investigación para luego, en tercera instancia, dar a conocer los resultados obtenidos. Posteriormente se discutirán los resultados, con relación a los antecedentes propuestos para, finalmente, presentar las conclusiones de esta investigación.

\section{Antecedentes teóricos}

La escritura y la alfabetización académica son fundamentales para el desarrollo del conocimiento en las distintas culturas o comunidades, ya que nos permiten acceder a las prácticas de lenguaje y pensamiento propias delámbito académico (Carlino, 2009). En este sentido, los estudios relacionados con la escritura académica han cobrado importancia en los últimos años (Cubo de Severino, 2005; Samraj, 2008; Swales, 1990, 2004), trayendo como consecuencia el desarrollo cada vez más sistematizado de los estudios alrededor de la práctica escritural que se realiza en las distintas comunidades discursivas, entendidas desde una perspectiva retórico-discursiva, como un grupo sociorretórico que agrupa miembros reunidos según grupos de ocupación o intereses especiales, desde donde emerge una práctica discursiva característica (Swales, 1990).

Desde esta óptica, en los estudios en torno a la escritura académica, interesan dos criterios de análisis de los géneros discursivos, fundamentados por Bajtín (1999), el estructural y el funcional, los que no se instancian por separado en los textos sino que se dan conjuntamente, idea que ha sido abordada desde una perspectiva psicosociodiscursiva de los estudios lingüísticos. Acerca de los estudios estructurales, estos han sido utilizados como un medio para explicar las características funcionales de los géneros académicos estudiados (Cubo de Severino, 2005; Parodi, 2008; Samraj, 2008; Swales, 1990). Es decir, el estudio de las estructuras que adoptan los diferentes géneros en el marco de la escritura académica contribuye a la descripción de los géneros y a una posible explicación de la funcionalidad de ellos en su contexto de producción y uso, desde un punto de vista sociodiscursivo (Parodi, 2008). 
En este sentido, la estructura y la organización funcional de un texto está indicada, con un mayor o menor grado de explicitud, por pistas léxico-gramaticales (Halliday, 1976). La interpretación de estas pistas léxico-gramaticales permitirá, por tanto, identificar la estructura de los textos así como los propósitos comunicativos que permiten reconocer que ciertos textos constituyen géneros textuales propios de una comunidad discursiva determinada (Askehave \& Swales, 2001).

\subsection{Enfoque estructural}

Herederos de la perspectiva estructuralista, los estudios contextualizados en este enfoque han buscado sistematizar las estructuras que subyacen a los diferentes textos. Desde ellos, se han estudiado secuencias, tipologías y macroestructuras textuales, siempre con el interés de clasificar y caracterizar los textos que nos rodean.

Bajo esta perspectiva, surge el concepto de superestructura, desde los planteamientos de Van Dijk (1978), entendida como la estructura formal que representa las partes en que se organiza el contenido de un texto. De este modo se transforma en un esqueleto caracterizador de los géneros discursivos, en donde cada uno de ellos posee una forma propia de ordenar la información, organizando las secuencias de frases con una función específica. Según la idea de Van Dijk (1978), la estructura de un texto desde un plano global se organiza en torno a dos estructuras: la macroestructura textual y la superestructura textual. La primera resume el contenido global del texto, mientras que la segunda representa la forma que adopta el discurso (Van Dijk, 1978).

Para este autor, en la superestructura, las frases de un texto se organizan por medio de un esquema que está constituido por categorías funcionales. Tal es el ejemplo de las noticias periodísticas, cuya superestructura se compone de un resumen, con titular y encabezamiento, y de un relato, con una situación y comentarios. Este esquema es transversal a todas las noticias sin importar los temas que pueda abordar (Van Dijk, 1978).

Las superestructuras tradicionalmente identificadas por Van Dijk (1978) vienen a conformar ciertos tipos textuales, tales como: la narración, la argumentación, el discurso periodístico y el informe experimental. Ante esto, Charadeau y Maingueneau (2005) establecen el inconveniente de aplicar la noción de superestructura, puesto que en ella se mezclan criterios como el modo en que el discurso se organiza, esto 
es, modalidades de organización discursiva (narración, descripción y argumentación) y géneros discursivos particulares (la noticia, el informe científico, etc.). Por otra parte, Adam $(1992,2005)$ aborda la problemática de la estructura de textos, planteando que la noción de prototipos de texto ha sido útil para redefinir la noción de superestructura de van Dijk (1987) y para distinguir dos dimensiones: la pragmática, referida a la intencionalidad, al marco de referencia de los enunciados y a la cohesión semántica global -equivalente a la macroestructura de Van Dijk (1987)- y la secuencial, referida a la gramática del texto -equivalente a la microestructura de Van Dijk (1987)- y a la organización de las diferentes secuencias del texto. Según Adam (1992, 2005), un texto puede estar estructurado en diferentes secuencias de base, dado que la posibilidad de que se estructure de manera única es muy baja. En todo caso, siempre existe un tipo de secuencia que destaca y que define la estructura dominante del texto. Para este, autor la secuencias posibles de identificar en un texto son las secuencias textuales, narrativa, argumentativa, explicativa, descriptiva y dialogal.

En cuanto al estudio de la estructura textual en escritura académica, Paltridge (2002) establece cuatro tipos de organización estructural con sus respectivos apartados para el género tesis, producido para acreditar conocimiento al término de un grado académico. Estas estructuras son:

- Tradicional Simple: compuesta por la estructura Introducción, Marco Teórico, Métodos, Resultados, Discusión y Conclusión;

- Tradicional Compleja: que consta de una Introducción y un Marco Teórico comunes y luego se plantean múltiples investigaciones individuales, con su respectivos apartados Introducción, Métodos, Resultados, Discusión y Conclusión, para finalizar con Discusión y Conclusión comunes;

- Basada en Tópico: que se compone por una Introducción, seguida de la revisión de un número variable de tópicos y finalizada por una Conclusión;

- Compilación de Artículos de Investigación: compuesta por una Introducción y un Marco Teórico comunes y luego se plantean múltiples artículos de investigación individuales, con su respectivos apartados Introducción, Marco Teórico, Métodos, Resultados, Discusión y Conclusión, para finalizar con una Conclusión común. 


\subsection{Enfoque funcional}

Los estudios enmarcados dentro del enfoque funcional han intentado establecer los roles y funciones desempeñados por los textos en sus contextos reales de uso (Swales, 1990, 2004; Bathia, 2002). En este sentido, la tarea ha sido caracterizar los textos y los géneros textuales, desde un paradigma sociodiscursivo, que responden a las necesidades de las comunidades discursivas en torno a la escritura de textos.

Sobre esta base emerge el concepto de Propósito Comunicativo (Swales, 1990, 2004; Askehave \& Swales, 2000) abordado como un concepto complejo que explica la intencionalidad con que se producen los textos según un contexto determinado. Para Askehave \& Swales (2000), una consecuencia inmediata de este enfoque funcional es que los géneros son abordados como eventos sociales comunicativos y no como meras categorías de discurso. De este modo, se intenta establecer una relación entre el propósito de un género y su estructura, lo que sugiere que la intención comunicativa del género es la que le da forma y le brinda una estructura esquemática interna. Lo anterior permitiría diferenciar dos géneros similares, pero que posean distintos propósitos.

Según la propuesta de los autores, las comunidades discursivas serían determinantes en la configuración de los propósitos comunicativos, pues, cada una de ellas presentará intencionalidades comunicativas propias de su área, necesarias de plasmar en los textos por medio de los propósitos comunicativos. De esta manera, en el ejercicio de clasificar un texto como parte de algún género, para Askehave \& Swales (2000), no basta con poner atención a las características estructurales sino también a la funcionalidad que demuestra la noción del propósito comunicativo, ya que este concepto resulta fundamental para la definición y constitución de los diversos géneros, académicos y no académicos.

Para finalizar, conviene destacar la noción de macropropósito, entendido como el propósito de un género, propuesta por Parodi, Venegas, Ibáñez y Gutiérrez (2008) para el género tesis, este es: "persuadir acerca de un planteamiento teórico o ideológico" (Parodi, Venegas, Ibáñez y Gutiérrez, 2008: 66). Esta idea nos acerca a nuestro objetivo de estudio, el cual es describir la estructura y propósitos comunicativos de los Trabajos Finales de Grado de Filosofía y Lingüística en los niveles de Licenciatura y Magíster, dando por supuesto que el macropropósito 
planteado alberga una serie de propósitos comunicativos asociados a la organización interna del género tesis.

\section{La investigación}

La presente investigación fue de tipo no experimental y de alcance descriptivo con un enfoque mixto, en donde se buscó caracterizar las variables determinadas para la investigación a partir de un análisis exhaustivo del corpus que permitió establecer categorías desde una búsqueda inductiva, basada en un modelo de etapas y fases de análisis con categorías emergentes, teniendo, como objetivo general, describir la estructura y propósitos comunicativos en los Trabajos Finales de Grado de Filosofía y Lingüística en los niveles de Licenciatura y Magíster.

\subsection{Corpus de investigación}

El corpus estuvo compuesto por 20 Tesis realizadas entre los años 2000 y 2009 en la Pontificia Universidad Católica de Valparaíso, Chile. Estas tesis pertenecen al Corpus TFGPUCV-2010 del proyecto Fondecyt 1101039 (Venegas, 2010). Las tesis corresponden a: cinco de Licenciatura en Lingüística, cinco de Licenciatura en Filosofía, cinco de Magíster en Lingüística Aplicada y cinco de Magíster en Filosofía. La elección de la muestra se realizó aleatoriamente a partir de los 407 textos que componen el corpus TFGPUCV-2010.

\subsection{Procedimientos generales}

El análisis se realizó en dos etapas. En la primera, se analizaron dos tesis de cada subgrupo, con el fin de identificar las estructuras y categorías léxico-gramaticales de los aparatados de cada tesis. Esto permitió construir una grilla de análisis que incluyó los criterios estructurales planteados por Paltridge (2002), categorías estructurales emergentes y pistas léxico-gramaticales, pertinentes para la identificación de los propósitos. Luego, se realizó la segunda etapa de análisis, en la cual se estudió el resto de las tesis de cada subgrupo.

\section{Resultados}

Los resultados obtenidos con relación a la estructura y sus apartados en las tesis según cada disciplina y nivel serán presentados mediante las siguientes tablas, ordenadas según el grado y el nivel al cual pertenecen. 


\subsection{Superestructura}

En el caso de las tesis de Licenciatura en Lingüística, se observa que, en los textos estudiados, existe un modelo más o menos regular para la conformación de su estructura. Todos las tesis presentaron el apartado Introducción. El apartado Marco Teórico se presentó en todas las tesis, pero con variantes, puesto que en la distinción entre Antecedentes y Marco de Referencia se presentaron diferencias. Así en una tesis se establece la distinción entre ambos subapartados; en otra todo el apartado Marco Teórico se denomina como Antecedentes; y en las tres restantes se utiliza la nomenclatura Marco Teórico sin hacer distinciones. El apartado Metodología estuvo presente en todas las tesis analizadas, pese a utilizarse etiquetas distintas, tales como Marco Metodológico y La Investigación. El apartado Resultados y Discusión también presentó algunas leves variantes, como Análisis de Resultados e Interpretación, sin embargo, tuvo lugar en todas las tesis. Lo mismo ocurrió con el apartado Conclusión que se presenta en todas las tesis, con algunos matices de denominación, como por ejemplo, Comentarios Finales. Lo anterior se grafica en la siguiente tabla:

\begin{tabular}{|l|c|c|c|c|c|}
\hline Apartados & TLinL_1 & TLinL_2 & TLinL_3 & TLinL_4 & TLinL_5 \\
\hline Introducción & $\mathrm{x}$ & $\mathrm{x}$ & $\mathrm{x}$ & $\mathrm{x}$ & $\mathrm{x}$ \\
\hline Antecedentes & $\mathrm{x}$ & & & $\mathrm{x}$ & \\
\hline Marco Teórico & $\mathrm{x}$ & $\mathrm{x}$ & $\mathrm{x}$ & & $\mathrm{x}$ \\
\hline Metodología & $\mathrm{x}$ & $\mathrm{x}$ & $\mathrm{x}$ & $\mathrm{x}$ & $\mathrm{x}$ \\
\hline $\begin{array}{l}\text { Resultados y } \\
\text { Discusión }\end{array}$ & $\mathrm{x}$ & $\mathrm{x}$ & $\mathrm{x}$ & $\mathrm{x}$ & $\mathrm{x}$ \\
\hline Conclusión & $\mathrm{x}$ & $\mathrm{x}$ & $\mathrm{x}$ & $\mathrm{x}$ & $\mathrm{x}$ \\
\hline
\end{tabular}

Tabla 1: Superestructura Lingüística Licenciatura

En cuanto a las tesis de Magíster en Lingüística, la estructura en los textos analizados sigue un modelo regular -Introducción, Marco Teórico, Metodología, Resultados y Discusión, y Conclusión- con algunas variantes en el modo de denominar los apartados, al igual como se explicó para el caso anterior de Licenciatura. Esto queda explicado en forma gráfica, en la tabla que se presenta a continuación: 


\begin{tabular}{|l|c|c|c|c|c|}
\hline Apartados & TLinM_1 & TLinM_2 & TLinM_3 & TLinM_4 & TLinM_5 \\
\hline Introducción & $\mathrm{x}$ & $\mathrm{x}$ & $\mathrm{x}$ & $\mathrm{x}$ & $\mathrm{x}$ \\
\hline Marco Teórico & $\mathrm{x}$ & $\mathrm{x}$ & $\mathrm{x}$ & $\mathrm{x}$ & $\mathrm{x}$ \\
\hline Metodología & $\mathrm{x}$ & $\mathrm{x}$ & $\mathrm{x}$ & $\mathrm{x}$ & $\mathrm{x}$ \\
\hline $\begin{array}{l}\text { Resultados y } \\
\text { Discusión }\end{array}$ & $\mathrm{x}$ & $\mathrm{x}$ & $\mathrm{x}$ & $\mathrm{x}$ & $\mathrm{x}$ \\
\hline Conclusión & $\mathrm{x}$ & $\mathrm{x}$ & $\mathrm{x}$ & $\mathrm{x}$ & $\mathrm{x}$ \\
\hline
\end{tabular}

Tabla 2: Superestructura Lingüística Magíster

Para el caso de Licenciatura en Filosofía, la superestructura de las tesis analizadas es un tanto más irregular que en las anteriores, aunque es posible establecer un patrón de la presencia de Introducción, una cierta cantidad de Capítulos que componen el desarrollo de la tesis, y finalmente una Conclusión. Así, dos de estas tesis presentaron tres Capítulos, uno presentó cuatro, uno presentó dos y, finalmente, uno presentó solamente un Capítulo pero sumado a la existencia de un Prólogo, lo que se presenta en la Tabla 3:

\begin{tabular}{|l|l|l|l|l|l|}
\hline Apartados & TFilL_1 & TFilL_2 & TFilL_3 & TFilL_4 & TFilL_5 \\
\hline Prólogo & & $\mathrm{x}$ & & & \\
\hline Introducción & $\mathrm{x}$ & $\mathrm{x}$ & $\mathrm{x}$ & $\mathrm{x}$ & $\mathrm{x}$ \\
\hline Capítulo 1 & $\mathrm{x}$ & $\mathrm{x}$ & $\mathrm{x}$ & $\mathrm{x}$ & $\mathrm{x}$ \\
\hline Capítulo 2 & $\mathrm{x}$ & & $\mathrm{x}$ & $\mathrm{x}$ & $\mathrm{x}$ \\
\hline Capítulo 3 & & & $\mathrm{x}$ & $\mathrm{x}$ & $\mathrm{x}$ \\
\hline Capítulo 4 & & & $\mathrm{x}$ & & \\
\hline Conclusión & $\mathrm{x}$ & $\mathrm{x}$ & $\mathrm{x}$ & $\mathrm{x}$ & $\mathrm{x}$ \\
\hline
\end{tabular}

Tabla 3: Superestructura Filosofía Licenciatura

Con relación a la estructura de las tesis de Magíster en Filosofía estudiadas, se presenta una regularidad en la mayoría de los textos analizados, por medio de la presencia de una Introducción, tres Capítulos que desarrollan el tema de la tesis y, finalmente, una Conclusión. Solo una tesis no presentó esta estructura al contener una Introducción y seis Capítulos, en ausencia de Conclusión (ver Tabla 4). 


\begin{tabular}{|l|c|c|c|c|c|}
\hline Apartados & TFilM_1 & TFilM_2 & TFilM_3 & TFilM_4 & TFilM_5 \\
\hline Introducción & $\mathrm{x}$ & $\mathrm{x}$ & $\mathrm{x}$ & $\mathrm{x}$ & $\mathrm{x}$ \\
\hline Capítulo 1 & $\mathrm{x}$ & $\mathrm{x}$ & $\mathrm{x}$ & $\mathrm{x}$ & $\mathrm{x}$ \\
\hline Capítulo 2 & $\mathrm{x}$ & $\mathbf{x}$ & $\mathrm{x}$ & $\mathrm{x}$ & $\mathrm{x}$ \\
\hline Capítulo 3 & $\mathbf{x}$ & $\mathbf{x}$ & $\mathrm{x}$ & $\mathrm{x}$ & $\mathrm{x}$ \\
\hline Capítulo 4 & $\mathbf{x}$ & & & & \\
\hline Capítulo 5 & $\mathbf{x}$ & & & & \\
\hline Capítulo 6 & $\mathbf{x}$ & & & & \\
\hline Conclusión & & $\mathrm{x}$ & $\mathrm{x}$ & $\mathrm{x}$ & $\mathrm{x}$ \\
\hline
\end{tabular}

Tabla 4: Superestructura Filosofía Magíster

\subsection{Propósitos comunicativos}

En el proceso de establecimiento de las definiciones emergentes para cada propósito, se advirtieron dos maneras de aproximación a los propósitos comunicativos. Estas tienen que ver con el modo en que el propósito comunicativo se evidencia en el texto, que puede estar explícito o implícito en el texto. Así, nos encontramos ante tesis cuyos propósitos comunicativos se encontraban explicitados al comienzo de cada apartado, o bien, en la introducción; y ante otras cuyos propósitos se encontraban implícitos y debieron inferirse a partir de las pistas léxico-gramaticales de los textos. Esto nos permitió sistematizar una continuidad entre aquellos propósitos más o menos explícitos que se presentaron en las tesis del corpus estudiado según cada disciplina y nivel.

En primer lugar, los propósitos comunicativos vinculados a las tesis de Magíster en Lingüística resultaron tener el mayor grado de explicitud, como se explica en el Esquema 1. Los propósitos comunicativos fueron, en el apartado Introducción, presentar el tema de investigación, el objetivo, los aspectos a indagar y las partes del trabajo, evidenciado únicamente mediante marcas implícitas. En el apartado Marco Teórico, se observaron marcas explícitas que llevaron a definir su propósito como exponer los antecedentes teóricos que sustentan la investigación. Por su parte, el propósito comunicativo asociado al apartado Metodología fue el de exponer los criterios metodológicos que sustentan la investigación, extraído a partir de marcas explícitas e implícitas, por medio del planteamiento del problema, pregunta de investigación, objetivo, 
corpus, variables. El propósito comunicativo del apartado Resultados y Discusión se evidenció como exponer los datos encontrados y proponer los análisis que sustenten la interpretación a través de marcas explícitas. Finalmente, en el apartado Conclusión, el propósito comunicativo identificado fue exponer una síntesis de los resultados y establecer limitaciones y proyecciones, cuyo hallazgo se caracterizó por la presencia de marcas explícitas e implícitas.

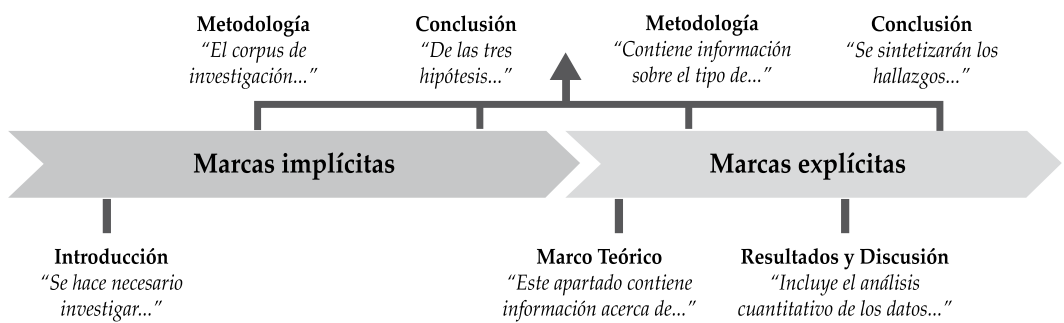

\section{Esquema 1: Propósitos Comunicativos en Magíster en Lingüística}

Los propósitos comunicativos asociados a las tesis de Lingüística en el nivel de Licenciatura resultaron en un nivel intermedio de explicitud, tal como se muestra en el Esquema 2. En el apartado Introducción, el propósito comunicativo a partir del corpus revisado fue presentar el tema de investigación, el objetivo, los aspectos a indagar y las partes del trabajo. Ello se obtuvo a partir de marcas implícitas. En el apartado Marco Teórico, el propósito comunicativo constó de exponer los antecedentes teóricos que sustentan la investigación, evidenciado por medio de marcas explícitas e implícitas. El propósito comunicativo del apartado Metodología fue exponer los criterios metodológicos que sustentan la investigación, atisbado implícitamente - por medio de la presentación del tipo de investigación, sujetos, preguntas, hipótesis, corpus, etc. - y explícitamente. Para el apartado Resultados y Discusión, el propósito comunicativo se relacionó con la exposición de los datos encontrados y de un análisis sustentador de la interpretación, el cual fue señalado en forma explícita. En el apartado Conclusión, el propósito comunicativo persiguió exponer una síntesis de los resultados y establecer limitaciones y proyecciones del trabajo, elementos que se encontraron aludidos tanto implícita como explícitamente. 


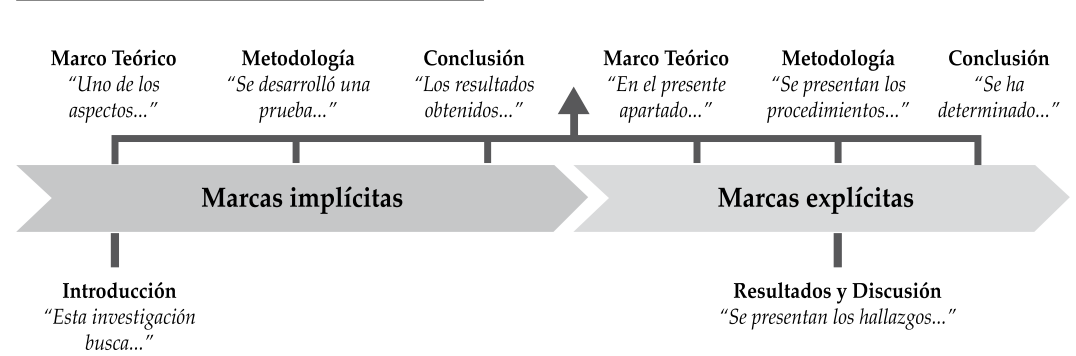

\section{Esquema 2: Propósitos Comunicativos en Licenciatura en Lingüística}

Otro conjunto de tesis que presentó sus propósitos comunicativos en un grado intermedio fue Filosofía en el nivel de Licenciatura, en donde fue posible establecer que, para el apartado Introducción, el propósito comunicativo que lo sustenta es presentar el tema de investigación, objetivo y aspectos a indagar, establecido a partir de marcas implícitas. Para el caso del apartado Capítulo, el propósito comunicativo está dado por marcas explícitas y/o implícitas y se conforma con exponer un tema acerca de la investigación. En el apartado Conclusión, el propósito comunicativo evidenciado fue exponer acerca de la síntesis y valoración de la investigación, manifestado por medio de marcas tanto explícitas como implícitas. Lo anterior se evidencia en el esquema 3:

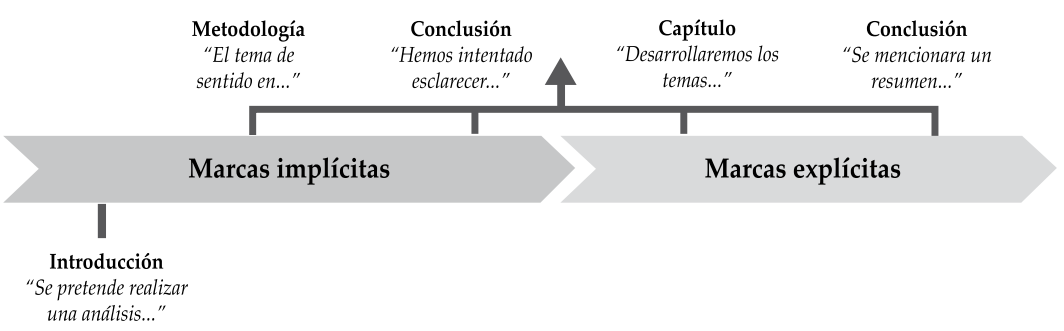

\section{Esquema 3: Propósitos Comunicativos en Licenciatura en Filosofía}

Acerca de los propósitos comunicativos que sustentan los apartados de las tesis de Magíster en Filosofía, fue posible constatar que, en el apartado Introducción, el propósito comunicativo está dado exclusivamente por marcas implícitas, tratamientos de temáticas, y es presentar el tema de investigación, el objetivo y los aspectos a indagar. En cuanto al apartado Capítulo, su propósito comunicativo está dado por marcas implícitas que dicen relación con el tratamiento de temáticas en profundidad y es exponer acerca de un tema de la investigación. Finalmente, el apartado Conclusión se caracteriza por sustentarse por un propósito comunicativo evidenciado también mediante marcas implícitas y que es exponer acerca de la síntesis y valoración final del trabajo. En este subgrupo, no se 
encontró ninguna huella explícita acerca de los propósitos comunicativos. Lo anterior se explica en el Esquema 4:

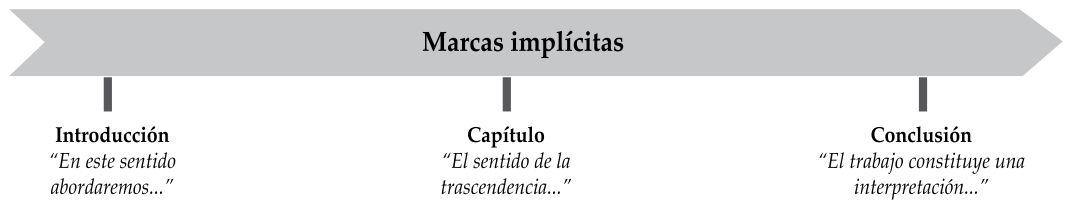

\section{Esquema 4: Propósitos Comunicativos en Magíster de Filosofía}

\section{Discusión}

A partir de los datos obtenidos, es posible establecer que, en cuanto a la comparación por disciplina, existe una estructura característica de cada una de las disciplinas estudiadas, Lingüística y Filosofía, lo que no ocurre en el caso de la diferenciación por nivel en donde no es posible distinguir una superestructura propia para cada uno sino que, en ambas disciplinas, la superestructura se vuelve común para ambos niveles estudiados, licenciatura y magíster.

De esta manera, la superestructura de las tesis de Lingüística está conformada por: Introducción, Marco Teórico, Metodología, Resultados y Conclusión; mientras que la de las tesis de Filosofía: Introducción, Capítulo(s) (uno o más, según sea el caso) y Conclusión. Esto plantea una diferencia fundamental entre ambas disciplinas ya que mientras en una el desarrollo de la tesis se realiza en tres apartados bien determinados, en la otra, el desarrollo no tiene una cantidad específica de apartados con un nombre establecido, sino que es más libre y varía en cada texto.

En relación a lo propuesto por Paltridge (2002), para la organización de las tesis, es posible afirmar que, en Lingüística, la estructura de las tesis del corpus estudiado se asemeja al tipo Tradicional Simple, pues presenta todos los apartados que le corresponden a este tipo de estructura. Esta organización, por su parte, se aleja ya de lo evidenciado para la superestructura de las tesis de Filosofía pues, como se mencionó anteriormente, en esta disciplina la estructura del trabajo final queda establecida por la presencia de una Introducción, un desarrollo por medio de Capítulo (s) y una Conclusión, lo que, siguiendo la idea de Paltridge (2002), correspondería una estructura de Tesis Basada en Tópico.

Lo anterior permite atisbar que las tesis de Lingüística se acercan más al género propiamente científico, debido a que los apartados 
que desarrolla siguen los principios de una investigación científica, de acuerdo a lo señalado por Swales (1990); mientras que las tesis de Filosofía presentan una exposición más bien descriptiva de los conceptos, basándose en un tópico articulador. A raíz de ello, fue posible encontrar diferencias disciplinares considerando las superestructuras propuestas y los planteamientos de Paltridge (2002).

Tomando como punto de partida la superestructura de las tesis de ambas disciplinas, arrojada de la primera parte de la investigación, se procedió a pesquisar los propósitos comunicativos articuladores de las superestructuras en cada uno de los apartados. Así, se obtuvo la configuración de los propósitos comunicativos para cada apartado de cada superestructura según la disciplina y nivel. Esto nos permitió darnos cuenta de que, tal como plantean Askehave \& Swales (2000), los propósitos comunicativos son capaces de hacer diferencias al interior de un mismo género. Esto es, ya que dentro del género tesis nos fue posible establecer la diferencia entre las disciplinas basándonos en el concepto de propósitos comunicativos. De este modo, los propósitos comunicativos en las tesis de Lingüística se encontraron orientados a sustentar teórica y metodológicamente una investigación empírica, así como también a desarrollar, defender y discutir esa investigación con relación a la teoría existente como un conocimiento nuevo. En cambio, en las tesis de Filosofía, los propósitos comunicativos estuvieron orientados a la exposición en torno a un tema, autor o libro, en donde todos los apartados se enfocaron hacia una descripción más que a una sustentación argumentativa, como lo fue en Lingüística. Por medio de lo anterior, los propósitos comunicativos permitieron establecer una diferencia de género según la disciplina abordada.

El fenómeno antes mencionado no ocurrió en el caso de la diferencia de nivel ya que, según lo expuesto en los resultados, no existieron diferencias significativas en los propósitos comunicativos de los apartados según el nivel al cual pertenecían, licenciatura o magíster, sino que más bien los propósitos se mantuvieron de acuerdo a la variable disciplinar.

Sin embargo, al llevar a cabo el procedimiento de marcaje en los textos del corpus, necesario para la identificación de los propósitos comunicativos, nos dimos cuenta de la existencia de dos modos de inserción de los propósitos dentro del texto, el modo explícito y el modo implícito, coherentemente con la propuesta de Askehave \& Swales (2000). 
Así, cuando el propósito es explícito, este tendió a ser mencionado al inicio de cada apartado, o bien en la introducción. Cuando es implícito, este debió ser inferido a partir de las pistas léxico-gramaticales y del contenido semántico de cada apartado. Este rasgo implicó una diferencia entre ambas disciplinas y niveles. De esta manera, en el caso de Lingüística se pudo evidenciar que, para el nivel de licenciatura, los modos de inserción de los propósitos comunicativos en el texto fueron explícitos e implícitos, mientras que, para el nivel de magíster, los modos en que los propósitos comunicativos fueron insertados en el texto resultaron ser más explícitos. Para el caso de Filosofía, en el nivel de licenciatura, los modos de inserción de los propósitos comunicativos en el texto fueron explícitos e implícitos, en tanto que en el nivel de magíster, los propósitos comunicativos se insertaron de un modo exclusivamente implícito. Lo anterior puede ser esquematizado en el siguiente continuum:

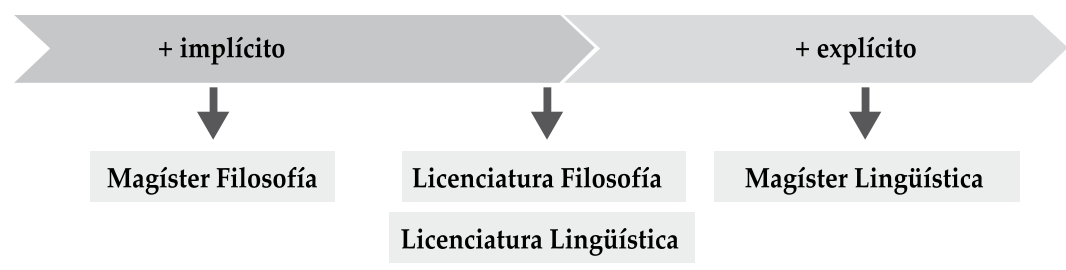

\section{Esquema 5 : Continuum grado de inserción del propósito comunicativo}

\section{Conclusiones}

A raíz de la investigación anteriormente planteada, se evidencia que, al analizar la superestructura, la variable que permitió establecer una diferencia entre las tesis analizadas fue la disciplina y en menor medida el nivel académico, es decir, la superestructura resultó ser similar según el nivel al cual pertenece, no así según la disciplina, en donde se obtuvieron superestructuras características de cada una de ellas. De esta manera, en Lingüística, la superestructura se compuso por: Introducción, Marco Teórico, Metodología, Resultados y Conclusión, respondiendo a la organización denominada Tradicional Simple, según Paltridge (2002). En tanto, en Filosofía, la superestructura constó de Introducción, Capítulos (uno o más, según sea el caso) y Conclusión, lo que la inscribe dentro de la estructura Basada en Tópico, propuesta por Paltridge (2002). 
En cuanto a los propósitos comunicativos, en Lingüística, estos se orientan a sustentar una investigación empírica, justificándola teórica y metodológicamente y, luego, discutiéndola como una vía de generación de conocimiento nuevo. En Filosofía, en cambio, los propósitos se centran en la exposición de contenido sobre algún tema, libro o autor. Además, con respecto al modo en que estos propósitos comunicativos se manifiestan en las tesis, se ha mostrado que a nivel de licenciatura en ambas disciplinas, ambos modos de identificación del propósito comunicativo, explícito e implícito, se dan en forma mixta. En tanto, en Magister de Filosofía, existe una significativa tendencia hacia la presencia de propósitos comunicativos implícitos, mientras que, en Magíster de Lingüística, se tiende a expresar los propósitos comunicativos con mayor grado de explicitud que en los otros subgrupos estudiados.

Los hallazgos presentados nos permiten concluir que, en Lingüística, existe una tendencia a textualizar una investigación, siguiendo un patrón compuesto por estructuras y propósitos bien definidos y aceptados por la comunidad discursiva de la disciplina. En tanto, en Filosofía, se observa una estructura de tipo muy general, es decir, reconocible en muchos otros géneros textuales (introducción, desarrollo, conclusión) y los propósitos, por lo general, se orientan a presentar una temática, exponer temas relacionados con ella y sintetizar las interpretaciones realizadas en cada capítulo. Estos hallazgos nos permiten evidenciar el hecho de que el género tesis no es homogéneo y que varía según las disciplinas, de acuerdo a las necesidades de las comunidades discursivas particulares.

Como se ha planteado al inicio, esta es una caracterización de las tesis en estudio, por lo que una limitación importante es que no es posible generalizar los resultados. En este sentido, proyectamos ampliar el corpus en disciplinas y niveles para obtener así una descripción más acabada de los fenómenos estudiados en la presente investigación. Otra proyección es ahondar en el estudio de los modos de inserción de los propósitos comunicativos en los textos, surgido en esta investigación. Lo anterior permitiría, posteriormente, generar aplicaciones didácticas para la escritura de tesis, que contengan la superestructura y propósitos comunicativos característicos, según la disciplina y el nivel, para así optimizar el grado de inserción en la comunidad discursiva de aquellos que escriben este género para acreditar su membresía en ciertas comunidades académicas. 


\section{Referencias bibliográficas}

Adam, J. (1992). Les textes. Types et prototypes. Paris: Nathan.

Adam, J. (2005) La linguistique textuelle. Introduction à l'analyse textuelle des discours. Paris: Armand Colin Éditeur.

Askehave, I. \& J. Swales (2001). Genre identification and communicative purpose: A problem and a possible solution. Applied Linguistics 22: 95-212.

Bhatia, V. (2002). Applied genre analysis: A multi-perspective model. En Ibérica 4, 3-19.

Carlino, P. (2009). Escribir, leer y aprender en la Universidad: Una introducción a la alfabetización académica [Online]. Disponible en: <http://www.relacionesdeltrabajo. fsoc.uba.ar/prod/alfabetizacion\%20academica. pdf $>$

Charaudeau, P. y D. Maingueneau (2005). Diccionario de análisis del discurso. Buenos Aires: Amorrortu.

Cubo de Severino, L. (2005). Los textos de la ciencia. Principales clases del discurso académico-científico. Córdoba: Comunicarte.

Halliday, M. (1976). Cohesion in English. Londres: Longman

Paltridge, B. (2002). Thesis and dissertation writing: An examination of published advice and actual practice. English for Specific Purposes 21: 125-143.

Parodi, G. (2008). Géneros del discurso escrito: Hacia una concepción integral desde una perspectiva sociocognitiva. En G. Parodi. (Ed.) Géneros académicos y géneros profesionales: Accesos discursivos para saber y hacer. Valparaíso: Ediciones Universitarias de Valparaíso.

Parodi, G., R. Venegas, R. Ibáñez y R. Gutiérrez (2008). Géneros del discurso en el Corpus PUCV-2006: Criterios, definiciones y ejemplos. En G. Parodi (Ed.), Géneros académicos y géneros profesionales: Accesos discursivos para saber y hacer. Valparaíso: Ediciones Universitarias de Valparaíso. 
Samraj, B. (2008). A discourse analysis of master's theses across disciplines with a focus on introductions. Journal of English for Academic Purposes 7: 55-67.

Swales, J. (1990). Genre analysis. English in academic and research settings. Cambridge: Cambridge University Press.

Swales, J. (2004). Research genres: Explorations and applications. Cambridge: Cambridge University Press.

Tamola, D. (2005). La tesina de licenciatura. En L. Cubo de Severino (Coord.), Los textos de la Ciencia. Principales clases del discurso académico-científico. 235-265). Córdoba: Comunicarte.

Van Dijk, T. A. (1978). La ciencia del texto. Un enfoque interdisciplinario. Barcelona: Paidós.

Venegas, R. (2010). Caracterización del macro-género trabajo final de grado en licenciatura y magíster: Desde los patrones léxico-gramaticales y retórico-estructurales al andamiaje de la escritura académica. Informe Proyecto FONDECYT Nº 1101039. 